\title{
The Clinical Efficacy of Nitrofurantoin for Treating Uncomplicated Urinary Tract Infection in Adults: A Systematic Review of Randomized Control Trials
}

\author{
Angelo Porreca $^{a}$ Daniele D'Agostino $^{b}$ Daniele Romagnoli ${ }^{b}$ \\ Francesco Del Giudice $^{c}$ Martina Maggi ${ }^{c}$ Katie Palmer ${ }^{d}$ Roberto Falabella ${ }^{e}$ \\ Ettore De Berardinis ${ }^{c}$ Alessandro Sciarrac ${ }^{c}$ Matteo Ferro ${ }^{f}$ Walter Artibani ${ }^{b}$ \\ Vincenzo Mirone $^{g}$ Gian Maria Busetto ${ }^{h}$

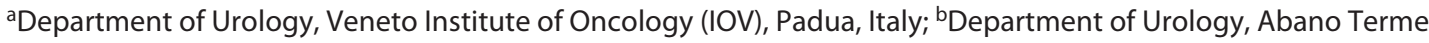 \\ Policlinic, Abano Terme, Italy; ' Department of Urology, Policlinico Umberto I, Sapienza University of Rome, \\ Rome, Italy; ${ }^{\mathrm{d} D e p a r t m e n t}$ of Internal Medicine and Geriatrics, Università Cattolica del Sacro Cuore, Rome, Italy; \\ eDepartment of Urology, San Carlo Hospital, Potenza, Italy; f'Department of Urology, IRCCS European Institute of \\ Oncology (IEO), Milan, Italy; ${ }^{9}$ Department of Urology, University of Naples Federico II, Naples, Italy; ${ }^{\text {h}}$ Department of \\ Urology and Renal Transplantation, University of Foggia Policlinico Riuniti of Foggia, Foggia, Italy
}

\section{Keywords}

Urinary tract infection - Uncomplicated urinary tract infection · Cystitis

\begin{abstract}
Objective: To provide an updated systematic review of randomized control trials (RCTs) to investigate the clinical and microbiological efficacy of nitrofurantoin compared to other antibiotics or placebo for treatment of uncomplicated urinary tract infections (uUTI). A secondary aim is to assess whether nitrofurantoin use is associated with increased side effects compared to other treatment regimens. Summary: The review was performed according to PRISMA guidelines. We searched 4 databases for articles published from database inception to May 6, 2020: (1) PubMed electronic database of the National Library of Medicine, (2) Web of Science, (3) Embase, and (4) Cochrane Library. Nine RCTs were selected for the review. RCTs were a mixture of double-blind, sin-
\end{abstract}

gle-blind, and open-label trials. The most common comparators were trimethoprim-sulfamethoxazole and fosfomycin tromethamine. Overall study quality was poor with a high risk of bias. The clinical cure rates in nitrofurantoin ranged from 51 to $94 \%$ depending on the length of follow-up, and bacteriological cure rates ranged from 61 to $92 \%$. Overall the evidence suggests that nitrofurantoin is at least comparable with other uUTI treatments in terms of efficacy. Patients taking nitrofurantoin reported fewer side effects than other drugs and the most commonly reported were gastrointestinal and central nervous system symptoms. Key Messages: Evidence on the clinical and bacteriological efficacy of nitrofurantoin is sparse, with a lack of new data, and hampered by high risk of bias. Although no firm conclusions can be made on the current base of evidence, the studies generally suggest that nitrofurantoin is at least comparable to other common uUTI treatments in terms of clinical and bacteriological cure. More robust research with well-designed double-blinded RCTs is needed. (c) 2021 S. Karger AG, Basel karger@karger.com

(C) 2021 S. Karger AG, Basel

www.karger.com/uin

Karger ${ }^{\prime}=$
Gian Maria Busetto

Department of Urology and Renal Transplantation

University of Foggia Policlinico Riuniti of Foggia

via Luigi Pinto, 1 - 71122 Foggia (Italy)

gianmaria.busetto@uniromal.it 


\section{Introduction}

Urinary tract infection (UTI) is a common disorder; by the age of 32 years, half of all women report having at least 1 previous UTI [1]. Most UTIs are defined as uncomplicated (uUTI), defined as UTI in a person who is not pregnant, is not immunocompromised, has no anatomical and functional abnormalities of the urogenital tract, and does not exhibit signs of tissue invasion and systemic infection [2]. Treatment options for UTI are becoming more limited due to increasing resistance to UTI antibiotics [3]. According to the updated guidelines by the Infectious Diseases Society of America and the European Society for Microbiology and Infectious Diseases [4], the first-line agents recommended for treating uUTI are nitrofurantoin monohydrate/macrocrystals and trimethoprim-sulfamethoxazole, although the latter was noted to have inferior efficacy compared with standard short-course regimens.

Nitrofurantoin has been used as a treatment for uUTI since the 1950s. However, the recommended regimen is 7 days, which has hampered its popularity in comparison to shorter or single-dose regimens, and there has been concern about resistance, but if given for only 3 days, nitrofurantoin's clinical efficacy diminishes [5]. Susceptibility to nitrofurantoin is reached if organisms' minimum inhibitory concentration is $32 \mu \mathrm{g} / \mathrm{mL}$ or less. Nitrofurantoin bioavailability is about $90 \%$ with $40 \%$ of urinary excretion. The drug is excreted in the urine achieving levels of $200 \mu \mathrm{g} / \mathrm{mL}$ or more. At a concentration $>100 \mu \mathrm{g} / \mathrm{mL}$, nitrofurantoin is a bactericide while it is bacteriostatic against organisms at concentrations $<32 \mu \mathrm{g} / \mathrm{mL}$ [6].

A systematic review in 2015 [5] concluded that nitrofurantoin toxicity is mild and predominantly gastrointestinal with low resistance rates. In a large study in Canada and European countries [7] resistance to nitrofurantoin was low in Escherichia coli (E Coli), $<3 \%$, while rates were higher with ampicillin (30\%), sulphonamides (29\%), trimethoprim (15\%), and trimethoprim/sulfamethoxazole (14\%). Nitrofurantoin has also shown to be effective in the prevention of UTIs [8]. An earlier review concluded that nitrofurantoin has an overall equivalence to trimethoprim/ sulfamethoxazole, ciprofloxacin, and amoxicillin [5] for the treatment of uUTI, but an update on the literature is needed. This is particularly important because there is low concordance with clinical guidelines for uUTI in primary care [9], with fluoroquinolones being the most commonly prescribed antibiotic in more than half of cases, and a significant trend toward increasing trimethoprim-sulfamethoxazole use and decreasing nitrofurantoin use.
The aim of the current paper is to provide an updated systematic review of randomized control trials (RCTs) to investigate the clinical and microbiological efficacy of nitrofurantoin compared to other antibiotics or placebo. A secondary aim is to assess whether nitrofurantoin use is associated with increased side effects compared to other treatment regimens.

\section{Systematic Review Procedure and Inclusion Criteria}

The review was carried out in accordance with the PRISMA recommendations [10]. PICOS was used to define the research question, as follows: (i) population - adults with uncomplicated UTI aged over 18; (ii) intervention - treatment with nitrofurantoin for UTI (monotherapy only); (iii) control/comparison - other antibiotics or placebo; (iv) outcomes - clinical efficacy and/or bacteriological cure (primary outcomes) and adverse effects (secondary outcome); (v) study design - RCT. Only studies in English were included.

\section{Database Search}

We searched 4 databases for articles published from database inception to May 6, 2020: (1) PubMed electronic database of the National Library of Medicine, (2) Web of Science, (3) Embase and, (4) Cochrane Library. MeSH terms and free words referring to the drug and $\mathrm{uUTI}$ were decided by 2 Urologists including the following key words: UTI, cystitis, bladder infection, urethritis, uUTI, urinary tract infection, acute cystitis, uncomplicated UTI, nitrofurantoin, Macrodantin, and Furadantin.

Two assessors independently screened the titles and abstracts of the selected studies according to the inclusion criteria defined in the PICOS, see above. The full texts of the articles selected by 1 or more of the assessors were retrieved for evaluation. Two assessors independently read the full texts and extracted the information from the selected studies. A third assessor reviewed the data extraction, and any disagreement was resolved through consensus. The numbers of abstracts screened, and studies assessed for eligibility, with reasons for exclusions at each stage, are presented in Figure 1.

\section{Data Extraction}

A data extraction form was designed to collect data relevant only to the aims of the current review, including study author and year, age and sex of participants, study design (open label, single or double blinded), identification of UTI at study inclusion (clinical symptoms and/or bacteriological confirmation), dosage of nitrofurantoin, comparator (placebo or other antibiotic) and dose, follow-up time(s), definition of 2 primary outcomes (symptomatic/clinical cure and bacteriological cure), and 1 secondary outcome (side/adverse effects), and results.

\section{Risk of Bias}

We used the Cochrane Collaboration's tool to assess risk of bias. Two authors independently assessed the quality of studies. Any disagreement was resolved via consensus discussion. 


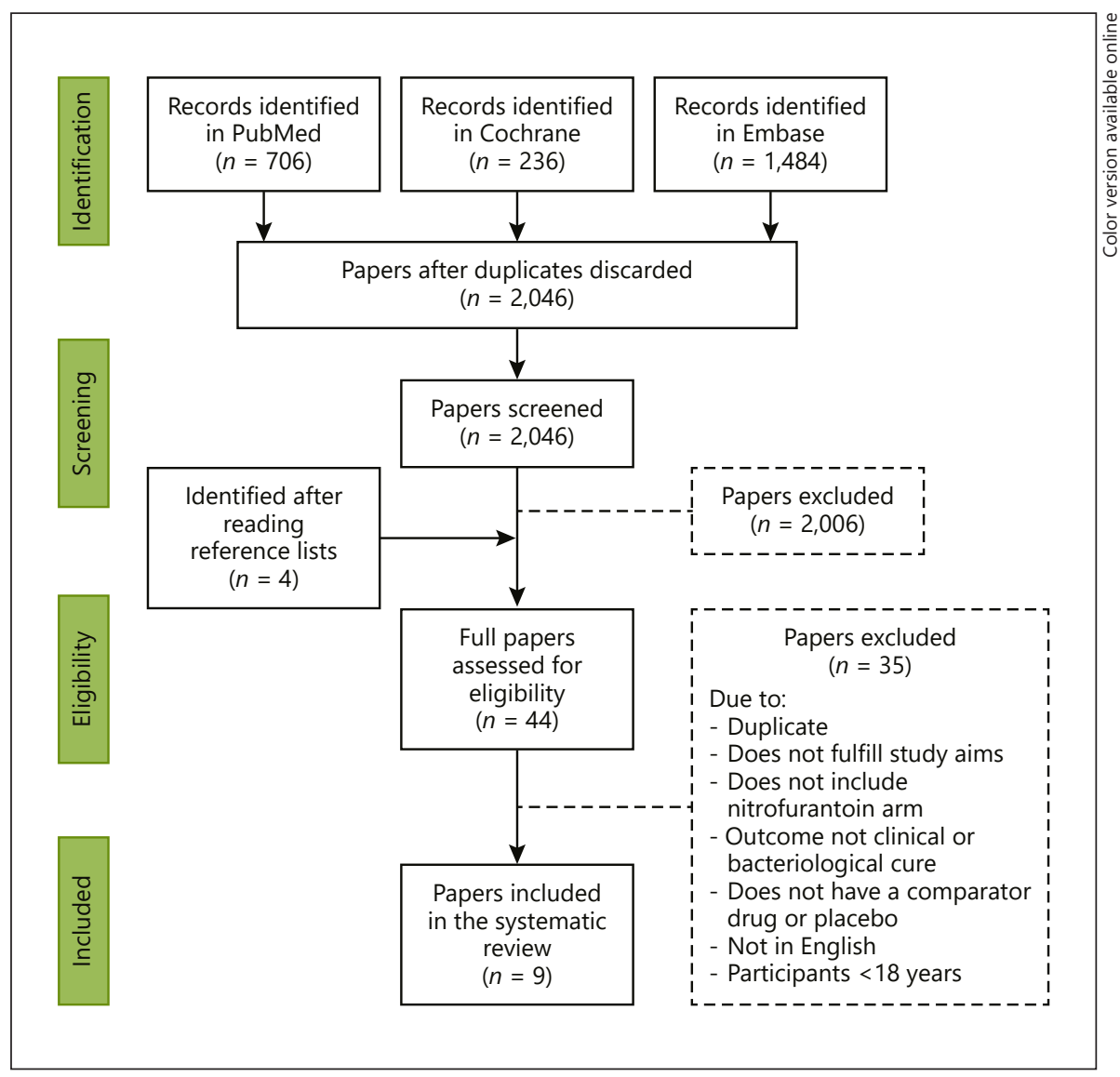

Fig. 1. PRISMA flowchart.

\section{Results}

\section{Search Results}

Figure 1 shows the PRISMA flowchart, including the key words used in the search strategies, the number of papers identified in the 4 databases, and the number and reason for exclusion. After removing duplicates, 2,046 titles and abstracts were screened; 44 were selected for fulltext reading and 35 were excluded because they did not match the aims of the current review.

\section{Study Characteristics}

Nine RCTs were selected for the review. Characteristics of the studies are detailed in Table 1. All the studies included only women. Four were double-blind RCTs [11-14], 1 observer blinded [15], and 3 open labels [1618], while 1 [19] did not state blinding status. Two studies $[16,19]$ included bacteriological resolution as an outcome (with or without symptomatic improvement), whereas the remaining studies all included 2 outcomes measures (clinical cure and bacteriological resolution). There were differences in the threshold for defining bacteriological cure, with 3 studies using $<10^{5} \mathrm{CFU} / \mathrm{mL}$ as a cutoff $[11,13,14], 1$ using $<10^{7} \mathrm{CFU} / \mathrm{mL}[18]$, and 4 using either $<10^{3} \mathrm{CFU} / \mathrm{mL}$ or $<10^{2} \mathrm{CFU} / \mathrm{mL}[12,16,17,19]$. Ludwig and Pauthner [15] did not specify the threshold. Follow-up times differed between studies, from 3 days to 6 weeks, with most studies having $>1$ follow-up points. All studies reported data on adverse effects and side effects.

One study [11] compared nitrofurantoin with a placebo while 5 had 1 comparator drug [12-16], and 3 compared with 2 or more other antibiotics $[12,18,19]$. The most common comparator was trimethoprim-sulfamethoxazole (co-trimoxazole) which was investigated in 4 studies $[12,16,18,19]$ and fosfomycin tromethamine, which was the comparator in 3 studies $[13,14,17]$. Oral ciprofloxacin [12], trimethoprim [18], cefadroxil [19], amoxicillin [19], and ofloxacin [15] were investigated in 1 study each. 


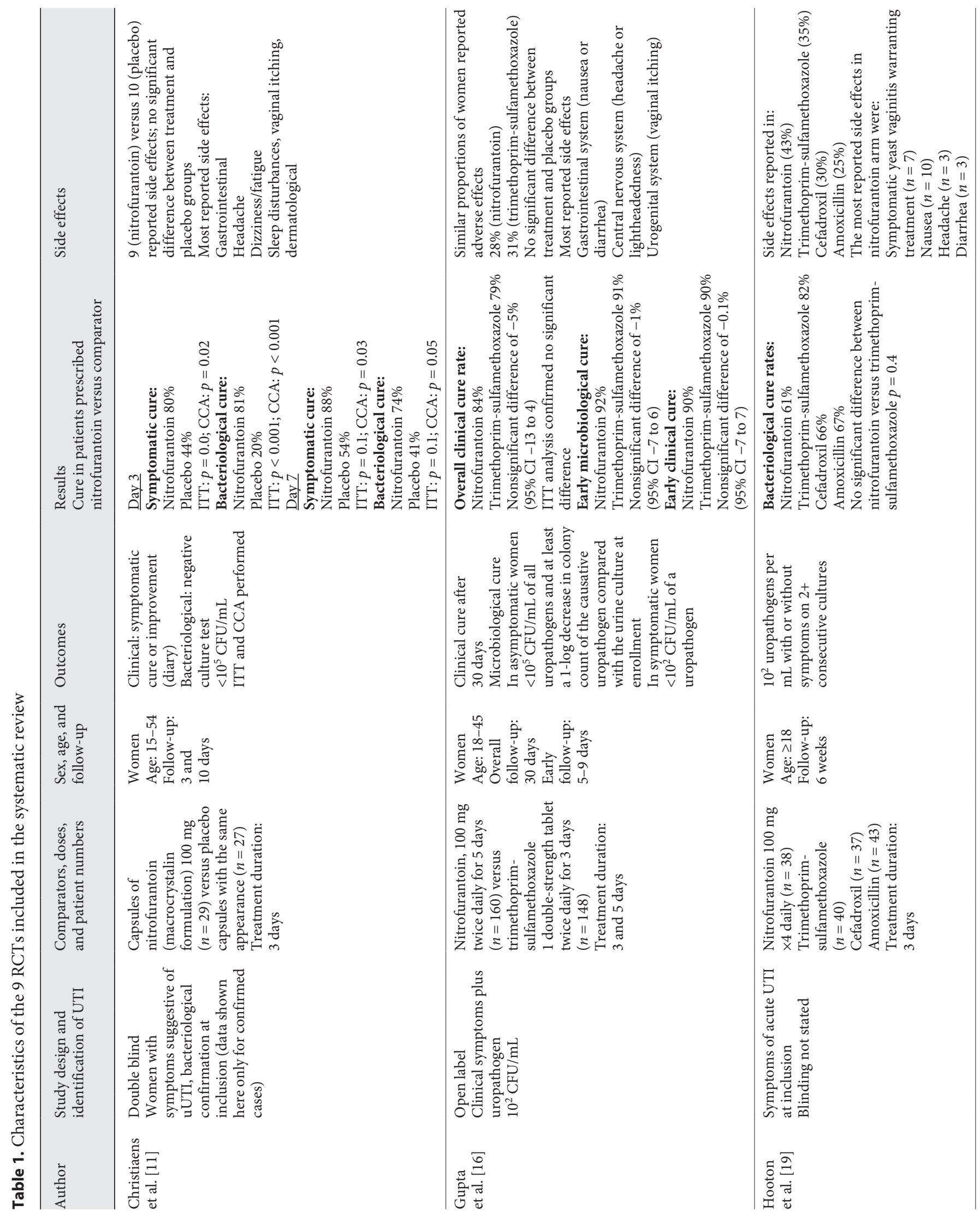



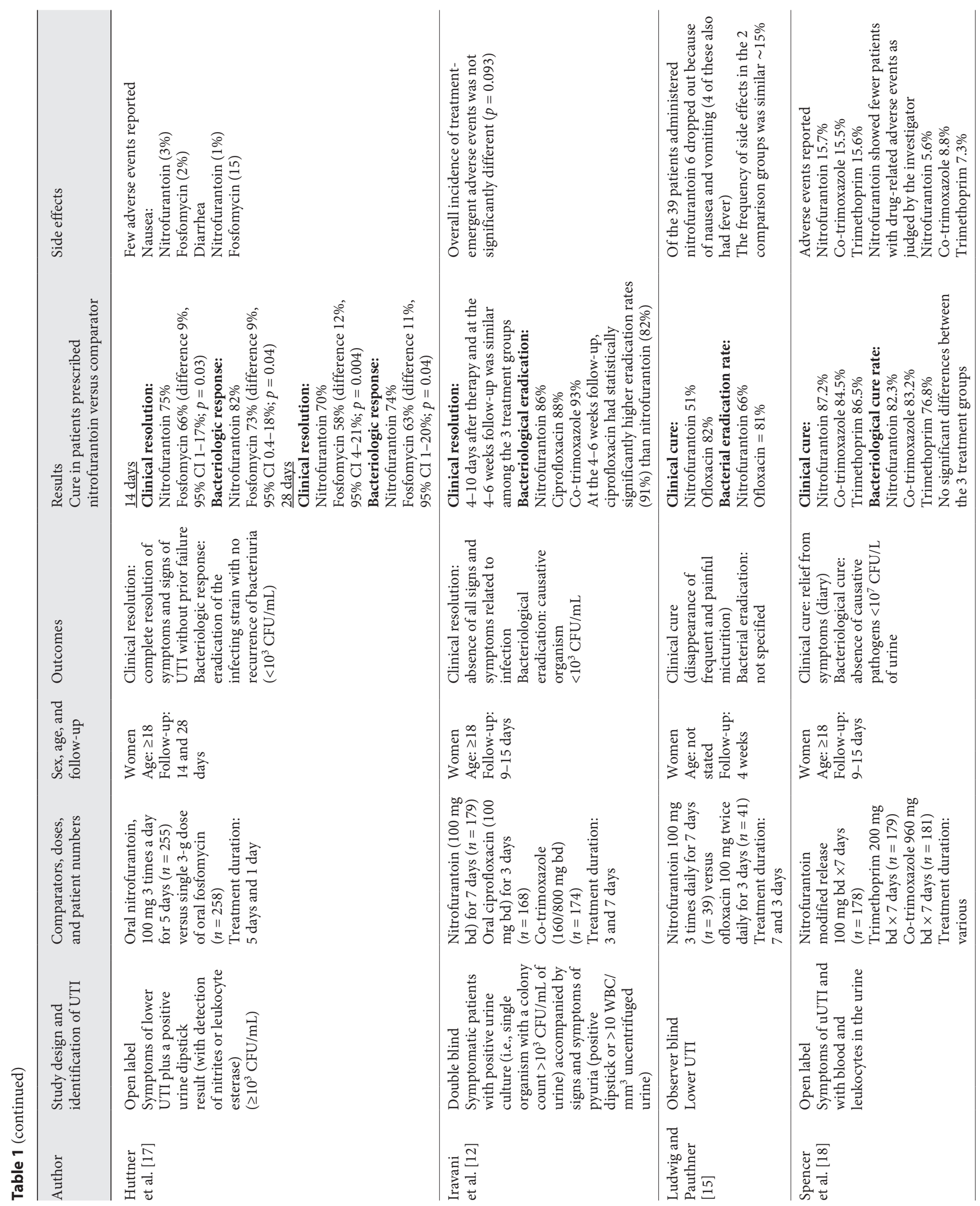

을을

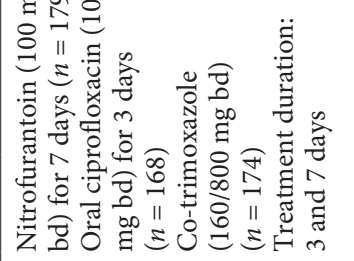

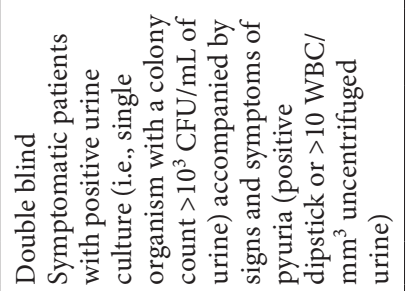

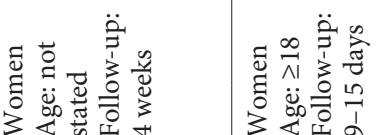

品爱厈

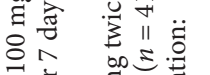
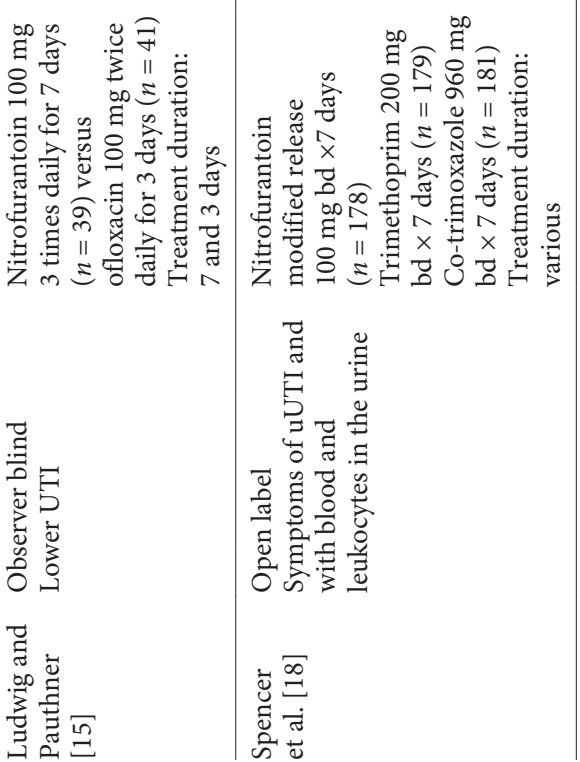


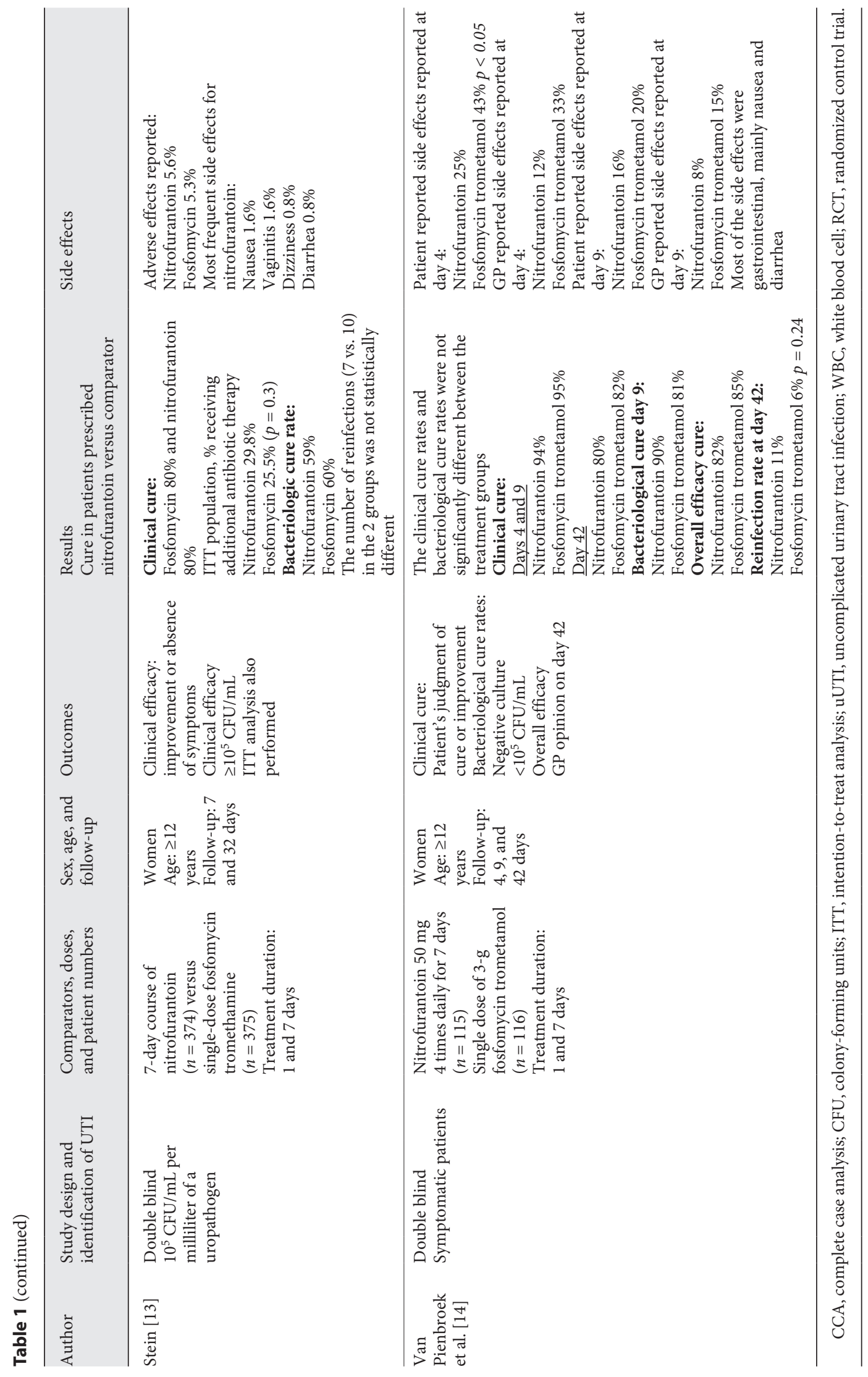


Table 2. Risk of bias ratings

\begin{tabular}{|c|c|c|c|c|c|c|c|}
\hline Author & $\begin{array}{l}\text { Random } \\
\text { sequence } \\
\text { (selection bias) }\end{array}$ & $\begin{array}{l}\text { Generation } \\
\text { allocation } \\
\text { Concealment } \\
\text { (selection bias) }\end{array}$ & $\begin{array}{l}\text { Blinding of } \\
\text { participants/ } \\
\text { personnel } \\
\text { (performance bias) }\end{array}$ & $\begin{array}{l}\text { Blinding of } \\
\text { outcomes } \\
\text { Assessment } \\
\text { (detection bias) }\end{array}$ & $\begin{array}{l}\text { Incomplete } \\
\text { outcome } \\
\text { Data (attrition } \\
\text { bias) }\end{array}$ & $\begin{array}{l}\text { Selective } \\
\text { reporting } \\
\text { (reporting } \\
\text { bias) }\end{array}$ & $\begin{array}{l}\text { Other } \\
\text { sources of } \\
\text { bias }\end{array}$ \\
\hline Christiaens et al. [11] & + & + & + & + & - & + & na \\
\hline Gupta et al. [16] & + & + & - & + & + & + & na \\
\hline Hooton et al. [19] & + & $?$ & - & + & - & + & na \\
\hline Huttner et al. [17] & + & + & - & + & + & + & na \\
\hline Iravani et al. [12] & + & + & + & + & + & + & na \\
\hline Ludwig and Pauthner [15] & + & $?$ & - & $?$ & - & $?$ & na \\
\hline Spencer et al. [18] & + & $?$ & - & + & + & + & na \\
\hline Stein [13] & + & $?$ & + & + & - & + & na \\
\hline Van Pienbroek et al. [14] & + & - & + & + & + & + & na \\
\hline
\end{tabular}

- High risk of bias; + low risk of bias; ? unclear risk of boas.

\section{Symptomatic/Clinical Cure}

The clinical cure rates in nitrofurantoin ranged from 51 [15] to 94\% [14] depending on the length of follow-up. The placebo-controlled trial showed a significantly higher clinical cure rate in patients treated with nitrofurantoin [11]. Two studies demonstrated a significantly higher clinical cure rate in patients treated with nitrofurantoin versus fosfomycin [13,17] whereas 1 found no difference [14]. Two studies reported no significant difference between nitrofurantoin and trimethoprim-sulfamethoxazole $[12,16]$. No significant difference between nitrofurantoin and oral ciprofloxacin was found [12]. Ludwig and Pauthner [15] reported that ofloxacin was superior to nitrofurantoin though no statistical test was performed, and it was noted that many nitrofurantoin patients discontinued because of side effects.

\section{Bacteriological Cure}

The bacteriological cure rates ranged from 61 [19] to $92 \%$ [16] depending on the length of follow-up. The placebo-controlled trial showed a significantly higher bacteriological cure rate in patients treated with nitrofurantoin [11]. Huttner et al. [17] demonstrated a significantly higher bacteriological cure rate in patients treated with nitrofurantoin versus fosfomycin (both at 14 and 28 days), whereas 2 found no significant difference $[13,14]$. Three studies found no significant difference between nitrofurantoin and trimethoprim-sulfamethoxazole [16, $18,19]$. There were no differences between nitrofurantoin and cefadroxil [19], amoxicillin [19], or trimethoprim [18] in terms of bacteriological cure. At a 4-6 week follow-up, Iravani et al. [12] reported that ciprofloxacin had statistically significantly higher eradication rates than nitrofurantoin.

\section{Side Effects}

Only one study reported higher side effects in patients taking nitrofurantoin compared to cefadroxil, amoxicillin, and trimethoprim-sulfamethoxazole [19]. Patients taking nitrofurantoin reported fewer side effects that those prescribed trimethoprim [18] or co-trimoxazole [18], or fosfomycin [14]. In the remaining studies, no differences in adverse events were reported between nitrofurantoin and placebo [11], trimethoprim-sulfamethoxazole [12, 16], ofloxacin [15], ciprofloxacin [12], or fosfomycin $[13,17]$. The most commonly reported side effects in patients taking nitrofurantoin were gastrointestinal (e.g., nausea or diarrhea) and central nervous system (e.g., headache) symptoms.

\section{Risk of Bias}

Table 2 shows results of the risk of bias assessment. Five studies were rated as having a high risk of bias due to lack of blinding, and general allocation (concealment) was unclear in 4 studies. For the bacteriological cure outcome, the detection bias was rated as low for all studies because they used objective measures from urine tests.

\section{Conclusion}

Our systematic review found only a limited number of RCTs comparing the clinical and bacteriological cure rates of nitrofurantoin with placebo or other antibiotic agents. 
Most were old: 6 were conducted in the 1980s and 1990s, with only one study published in the last 10 years. Results were heterogeneous but overall, the studies suggest that nitrofurantoin is at least equivalent to other antibiotics for the clinical and bacteriological cure of uUTI, and in some studies, it was demonstrated to be superior to trimethoprim-sulfamethoxazole. The only agent demonstrated to have higher bacteriological cure rates than nitrofurantoin was ciprofloxacin, which was reported in 1 study. Most studies reported no significant differences in side effects between nitrofurantoin and other antibiotic agents. Generally, the studies had a high risk of bias, mostly due to lack of blinding or unclear concealment methods.

There were large differences in the methodology of the studies. In particular, the threshold for defining bacteriological cure varied enormously from $<10^{2}$ to $<10^{7} \mathrm{CFU} /$ $\mathrm{mL}$. FDA guidelines for patient eligibility for enrollment in uUTI trials state a threshold of $\geq 10^{5} \mathrm{CFU} / \mathrm{mL}$ in symptomatic adult and adolescent females [20]. Further, less than half of the studies were double-blind RCTs. It is also likely that there were variations in the definition of clinical cure although such a parameter was more difficult to determine as many studies did not specifically state their methodology for assessing this outcome.

The evidence suggested that nitrofurantoin is at least equivalent to other antibiotics for the clinical and bacteriological cure of uUTI in women, confirming results of previous meta-analyses $[5,21]$ Our review suggested that nitrofurantoin was not associated with a higher risk of side effects than other antibiotic agents. However, previous case reports have documented adverse effects in individual patients, including lung injuries such as organizing pneumonia [22], interstitial lung disease [23], respiratory arrest [24], and pleural effusion [25]. Another report highlighted a case of systemic inflammatory response syndrome in a 74-year-old woman [26]. Only one study in our review reported higher adverse effects of nitrofurantoin compared to cefadroxil, amoxicillin, and trimethoprim-sulfamethoxazole, and in remaining studies rates were equivalent, the most common being, nausea, headache, and diarrhea. The clinical efficacy and equivalence in adverse effects to other antibiotics provide support to the guidelines [4] that recommend nitrofurantoin as the first-line agent for treating uUTI.

The aim of our review was to investigate the overall clinical and bacteriological efficacy of nitrofurantoin, but it is worth noting that the efficacy can differ depending on the organism causing the uUTI. The most common causative organisms are E. coli [16], others include S. saprophyticus, Enterococci, Klebsiella species, and Proteus mira- bilis, etc. Evidence from the studies included in the current review also revealed some differences in the efficacy of nitrofurantoin for curing the different uropathogens. For example, in Huttner et al.'s [17] study, 57-56\% of uUTIs were due to E. coli. When looking in this subgroup of patients, they found that the clinical response to nitrofurantoin was significantly higher than fosfomycin, and more pronounced when all uUTIs, irrespectively of the uropathogen involved were analyzed. In Ludwig and Pauthner [15] trial, ofloxacin was better than nitrofurantoin at eliminating E. coli, whereas nitrofurantoin had better eradication rates for Enterococci. In Spencer et al.'s [18] RCT, co-trimoxazole, trimethoprim, and nitrofurantoin had equivalent efficacy against $E$. coli, but against all other organisms (including Staphylococcus pluralis, Klebsiella pluralis, Enterococci, etc.) nitrofurantoin and co-trimoxazole were almost $15 \%$ better than trimethoprim. However, in all studies E. coli accounted for the majority of uUTIs and, therefore, numbers were low for comparing the efficacy of different antibiotics for other uropathogens.

In the current review, we focused on 2 main outcomes: symptomatic cure and microbiological cure. However, there are many other endpoints that have clinical relevance to patients and health-care providers, for example, quality of life, or cost-effectiveness. An open-label RCT [27] suggested that patients experiencing a clinical cure had significantly better QoL than patients with failed treatment, but this was not dependent on the drug prescribed. Hooton et al. [19] included a cost analysis in their study based in USA and reported higher costs for nitrofurantoin (USD 155 per patient) compared to trimethoprim-sulfamethoxazole (USD 114), which were mainly due to higher drug and pharmacy charges (3-4 times as high) and costs associated with more frequent return visits to the clinic for treatment of recurrent UTI and/or yeast vaginitis. However, another study reported that cost-effectiveness differed depending on resistance rates. For example, when the threshold for resistance is set at $<30 \%$ trimethoprim is more cost-effective than nitrofurantoin, but at $\geq 35 \%$ nitrofurantoin has the higher costefficiency. Last update of the German clinical guideline on epidemiology, diagnostics, therapy, prevention, and management of uUTI reports as resistance is a growing global problem that leads to significant challenges and costs in the health-care system. An international reevaluation of therapeutic recommendations in UUTI is guided by the resistance level of pathogens against commonly used antibiotics and by cost increase $[28,29]$.

Our review highlighted some gaps in current knowledge. First, all of the trials included in our review were 
restricted to female participants. Generally, UTIs in men are considered to be complicated because the prostate is often involved [30], although in some rare circumstances they could be considered uncomplicated in young men with a UTI without systemic symptoms, where the patient's medical history and physical examination do not suggest a causative factor [2]. Our search did not find any RCTs on the efficacy of nitrofurantoin for treating uUTI in men. Second, most studies were old (conducted in the 1980s and 1990s), and few had a robust methodology with double blinding and adequate concealment methods. Third, most of the studies were not powered to investigate efficacy according to less common causative uropathogens, and therefore, most drug comparisons could only provide sufficiently powered results related to E. coli. Fourth, the comparator drugs differed hugely between studies, making difficult to perform a methodologically sound meta-analysis. The most common comparator was trimethoprim-sulfamethoxazole. Fifth, no association with compounds and dietary supplement was included as comparator or in association with antibiotics [31].

In conclusion, the current systematic review highlighted the need for more RCTs to investigate the efficacy of nitrofurantoin for treating uUTI. Studies are old, and risk of bias is generally high. Although no firm conclusions can be made based on the current base of evidence, the studies generally suggest that nitrofurantoin is at least comparable to other common uUTI treatments in terms of clinical and bacteriological cure. Furthermore, recent fluoroquinolone warning on side effects represents another reason to prefer other molecules to treat uUTI.

\section{Conflict of Interest Statement}

The authors have nothing to disclose.

\section{Funding Sources}

The authors did not receive any funding.

\section{Author Contributions}

Conception or design of the work: Matteo Ferro, Gian Maria Busetto, Katie Palmer, and Angelo Porreca. Acquisition of data: Gian Maria Busetto, Francesco Del Giudice, Martina Maggi, Daniele Romagnoli. Interpretation of data for the work: Angelo Porreca, Ettore De Berardinis, Alessandro Sciarra, Katie Palmer, and Daniele D'Agostino. Drafting the paper: Gian Maria Busetto, Daniele D'Agostino, Daniele Romagnoli, and Angelo Porreca. Paper critically revising: Ettore De Berardinis, Alessandro Sciarra, Vincenzo Mirone, Matteo Ferro, Walter Artibni, Roberto Falabelle, and Daniele D'Agostino.

\section{References}

1 Foxman B, Brown P. Epidemiology of urinary tract infections: transmission and risk factors, incidence, and costs. Infect Dis Clin North Am. 2003;17(2):227-41.

2 Geerlings SE. Clinical presentations and epidemiology of urinary tract infections. Microbiol Spectr. 2016;4(5).

3 Hooton TM, Besser R, Foxman B, Fritsche TR, Nicolle LE. Acute uncomplicated cystitis in an era of increasing antibiotic resistance: a proposed approach to empirical therapy. Clin Infect Dis. 2004;39(1):75-80.

4 Gupta K, Hooton TM, Naber KG, Wullt B, Colgan R, Miller LG, et al. International clinical practice guidelines for the treatment of acute uncomplicated cystitis and pyelonephritis in women: a 2010 update by the Infectious Diseases Society of America and the European Society for Microbiology and Infectious Diseases. Clin Infect Dis. 2011;52(5): e103-20.

5 Huttner A, Verhaegh EM, Harbarth S, Muller AE, Theuretzbacher U, Mouton JW. Nitrofurantoin revisited: a systematic review and meta-analysis of controlled trials. J Antimicrob Chemother. 2015;70(9):2456-64.
6 Shah RR, Wade G. Reappraisal of the risk/ benefit of nitrofurantoin: review of toxicity and efficacy. Adverse Drug React Acute Poisoning Rev. 1989;8(4):183-201.

7 Kahlmeter G. Prevalence and antimicrobial susceptibility of pathogens in uncomplicated cystitis in Europe. The ECO.SENS study. Int J Antimicrob Agents. 2003;22(Suppl 2):49-52.

8 Muller AE, Verhaegh EM, Harbarth S, Mouton JW, Huttner A. Nitrofurantoin's efficacy and safety as prophylaxis for urinary tract infections: a systematic review of the literature and meta-analysis of controlled trials. Clin Microbiol Infect. 2017;23(6):355-62.

9 Grigoryan L, Zoorob R, Wang H, Trautner BW. Low concordance with guidelines for treatment of acute cystitis in primary care. Open Forum Infect Dis. 2015;2(4):ofv159.

10 Liberati A, Altman DG, Tetzlaff J, Mulrow C, Gøtzsche PC, Ioannidis JP, et al. The PRISMA statement for reporting systematic reviews and meta-analyses of studies that evaluate healthcare interventions: explanation and elaboration. BMJ. 2009;339:b2700.
11 Christiaens TC, De Meyere M, Verschraegen G, Peersman W, Heytens S, De Maeseneer JM. Randomised controlled trial of nitrofurantoin versus placebo in the treatment of uncomplicated urinary tract infection in adult women. Br J Gen Pract. 2002;52(482): 729-34.

12 Iravani A, Klimberg I, Briefer C, Munera C, Kowalsky SF, Echols RM. A trial comparing low-dose, short-course ciprofloxacin and standard 7 day therapy with co-trimoxazole or nitrofurantoin in the treatment of uncomplicated urinary tract infection. J Antimicrob Chemother. 1999;43(Suppl A):67-75.

13 Stein GE. Comparison of single-dose fosfomycin and a 7-day course of nitrofurantoin in female patients with uncomplicated urinary tract infection. Clin Ther. 1999;21(11):186472 .

14 Van Pienbroek E, Hermans J, Kaptein AA, Mulder JD. Fosfomycin trometamol in a single dose versus seven days nitrofurantoin in the treatment of acute uncomplicated urinary tract infections in women. Pharm World Sci. 1993;15(6):257-62. 
15 Ludwig G, Pauthner H. Clinical experience with ofloxacin in upper and lower urinary tract infections. A comparison with co-trimoxazole and nitrofurantoin. Drugs. 1987; 34(Suppl 1):95-9.

16 Gupta K, Hooton TM, Roberts PL, Stamm WE. Short-course nitrofurantoin for the treatment of acute uncomplicated cystitis in women. Arch Intern Med. 2007;167(20): 2207-12.

17 Huttner A, Kowalczyk A, Turjeman A, Babich T, Brossier C, Eliakim-Raz N, et al. Effect of 5-day nitrofurantoin vs single-dose fosfomycin on clinical resolution of uncomplicated lower urinary tract infection in women: a randomized clinical trial. JAMA. 2018;319(17): 1781-9.

18 Spencer RC, Moseley DJ, Greensmith MJ. Nitrofurantoin modified release versus trimethoprim or co-trimoxazole in the treatment of uncomplicated urinary tract infection in general practice. J Antimicrob Chemother. 1994;33(Suppl A):121-9.

19 Hooton TM, Winter C, Tiu F, Stamm WE. Randomized comparative trial and cost analysis of 3-day antimicrobial regimens for treatment of acute cystitis in women. JAMA. 1995; 273(1):41-5.

20 Available from: https://www.fda.gov/regulatory-information/search-fda-guidance-documents/uncomplicated-urinary-tract-infections-developing-drugs-treatment-guidanceindustry. Accessed 2020 Aug 16.
21 Mitrani-Gold FS, Raychaudhuri A, Rao S. Systematic review and meta-analysis to estimate the antibacterial treatment effect of nitrofurantoin for a non-inferiority trial in uncomplicated urinary tract infection. J Glob Antimicrob Resist. 2020;22:68-77.

22 Almeida P, Seixas E, Pinheiro B, Ferreira P, Araújo A. Consider nitrofurantoin as a cause of lung injury. Eur J Case Rep Intern Med. 2019;6(11):1.

23 Chin AJ, Rashid S, Gharibeh TR, Kibbe PS, Wynbrandt JH. Interstitial lung disease secondary to long-term nitrofurantoin use. Am J Case Rep. 2020;21:e920386.

24 Coban H. Respiratory arrest due to nitrofurantoin treatment in the absence of pulmonary pathology. J Basic Clin Pharm. 2015; 6(4):115-6.

25 Davis JW, Jones LS. Pleural effusion: an uncommon manifestation of nitrofurantoin-induced pulmonary injury. Respir Med Case Rep. 2016;19:65-7.

26 Gandotra SD, Smotrys MA, Patel DB, Chadha A. Systemic inflammatory response syndrome (SIRS) and a left bundle branch block (LBBB) due to nitrofurantoin. BMJ Case Rep. 2017;2017:bcr2016218127.
27 Ernst EJ, Ernst ME, Hoehns JD, Bergus GR. Women's quality of life is decreased by acute cystitis and antibiotic adverse effects associated with treatment. Health Qual Life Outcomes. 2005;3:45.

28 Kranz J, Schmidt S, Lebert C, Schneidewind L, Mandraka F, Kunze M, et al. The 2017 update of the German clinical guideline on epidemiology, diagnostics, therapy, prevention, and management of uncomplicated urinary tract infections in adult patients: part 1. Urol Int 2018;100(3):263-70.

29 Kranz J, Schmidt S, Lebert C, Schneidewind L, Mandraka F, Kunze M, et al. The 2017 update of the German clinical guideline on epidemiology, diagnostics, therapy, prevention, and management of uncomplicated urinary tract infections in adult patients: part II: therapy and prevention. Urol Int. 2018;100(3):271-8.

30 Busetto GM, Giovannone R, Ferro M, Tricarico S, Del Giudice F, Matei DV, et al. Chronic bacterial prostatitis: efficacy of short-lasting antibiotic therapy with prulifloxacin (Unidrox ${ }^{\circledR}$ ) in association with saw palmetto extract, lactobacillus sporogens and arbutin (Lactorepens ${ }^{\circledR}$ ). BMC Urol. 2014 Jul;14:53.

31 Pugliese D, Acampora A, Porreca A, Schips L, Cindolo L. Effectiveness of a novel oral combination of D-Mannose, pomegranate extract, prebiotics and probiotics in the treatment of acute cystitis in women. Arch Ital Urol Androl. 2020 Apr 6;92(1):34-8. 